\title{
Nonlinear absorption measurements of aza- borondipyrromethene dyes by the Z-Scan method
}

\author{
Hao Jung Chang \\ CREOL, The College of Optics \\ and Photonics, University of \\ Central Florida \\ Orlando, FL 32816, USA \\ hjchang@,knights.ucf.edu \\ Oliver Maury \\ Univ. Lyon, ENS \\ CNRS, Université Lyon 1, \\ Laboratoire de Chimie \\ Lyon, France \\ olivier.maury@ens-lyon.fr
}

\author{
Sanaz Faryadras \\ CREOL, The College of Optics \\ and Photonics, University of \\ Central Florida \\ Orlando, FL 32816, USA \\ s.faryadras@knights.ucf.edu \\ Gerard Berginc \\ Thales Research \& Technology, \\ Route Départementale, 91120 \\ Palaiseau, France \\ Gerard.berginc@,fr.thalesgroup.c \\ om
}

\author{
Sepehr. Benis \\ CREOL, The College of Optics \\ and Photonics, University of \\ Central Florida \\ Orlando, FL 32816, USA \\ s.benis@creol.ucf.edu \\ Andraud Chantal \\ Univ. Lyon, ENS \\ CNRS, Université Lyon 1, \\ Laboratoire de Chimie \\ Lyon, France \\ chantal.andraud@ens-lyon.fr
}

\author{
Sylvain David \\ Univ. Lyon, ENS \\ CNRS, Université Lyon 1, \\ Laboratoire de Chimie \\ Lyon, France \\ sylvain.david@ens-lyon.fr \\ David J. Hagan \\ CREOL, The College of Optics \\ and Photonics, University of \\ Central Florida \\ Orlando, FL 32816, USA \\ Hagan@creol.ucf.edu
}

Eric W. Van Stryland

CREOL, The College of Optics and

Photonics, University of Central Florida

Orlando, FL 32816, USA

ewvs@creol.ucf.edu

\begin{abstract}
We studied the effects of bromine substitution on the nonlinear absorption properties of aza-dipyrromethene dyes. Nonlinear absorption is measured at wavelengths of 1200, 1500, and $1700 \mathrm{~nm}$. At 1200nm, excited-state absorption due to two-photon excitation appears, and it is more pronounced in the brominated polymethine cyanine.
\end{abstract}

Keywords-nonlinear optics, nonlinear optical materials, optical nonlinearities in organic materials, Aza-BODIPY

\section{INTRODUCTION}

Organic molecules have been widely studied for nonlinear optical applications such as optical limiting and all-optical switching. An ideal optical limiter should have high transmission for low irradiance light and low transmission for high irradiance. This effect can be implemented utilizing mechanisms such as nonlinear absorption (NLA), including two-photon absorption (2PA), reverse saturable absorption, nonlinear refraction, and nonlinear scattering[1]. Azadipyrromethenes dyes' (Aza-BODIPY) potential for optical power limiting (OPL) in the near infrared region has been reported [2], and their excellent chemical and physical stability enabled the use of this class of dyes for the first solid-state devices for OPL in this spectral range [3]. The OPL mechanism of Aza-BODIPY is triggered by 2PA but its efficiency strongly relies on the spectral overlap between 2PA and excited-state absorption (ESA) . We designed two new molecules and their halogenated counterparts to explore the 2PA/ESA overlap.

\section{EXPERIMENT AND RESULTS}

\section{A. Sample information}

We studied the effect of bromine atoms on the nonlinear absorption of aza-boron dipyrromethene (Aza-BODIPY), which is shown in Fig.1. SD249 and SD230 are brominated compounds of SD238 and SD190, respectively. The steadystate linear absorption spectra of these molecules are shown Fig.1(a,b). The spectra in Fig.1 are of the solution of molecules dissolved in dichloromethane (DCM).

In this work, we use the Z-Scan technique[4], which is a single beam methodology to measure the optical nonlinearities such as nonlinear absorption and nonlinear refraction simultaneously. In this technique the sample is placed on a motorized stage and translated along the focus. The irradiance changes as it focuses and defocuses, and hence it alters the nonlinear transmission of the sample. The recorded transmission gives temporally and spatially averaged nonlinear absorption. In this work, a regeneratively amplified Ti:sapphire

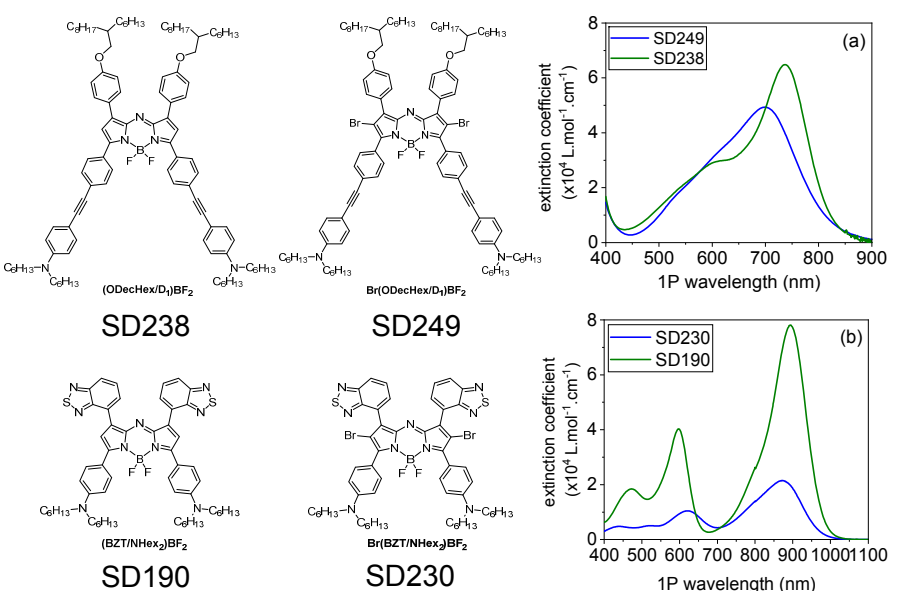

Fig.1. Extinction coefficient of aza-boron dipyrromethene ( $A z a-B O D I P Y)$ dyes, (a) SD249 and SD238, and (b) SD230 and SD190 with and without bromine at the side position. Molecular structure of each compound is shown on the left. 

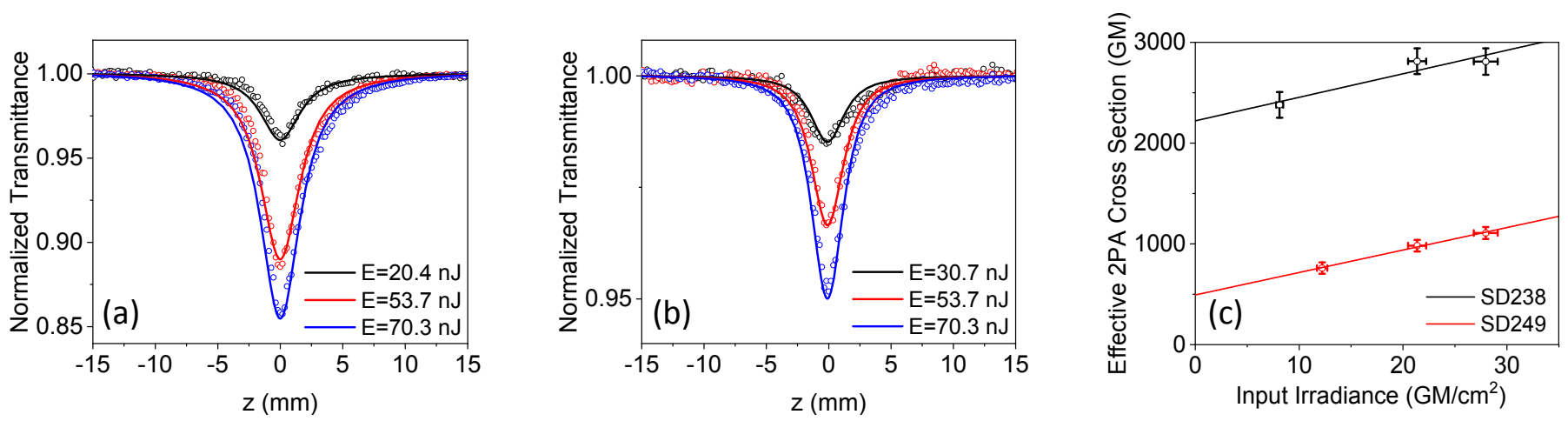

Fig.2. OA Z-Scan results of (a) SD238, (b) SD249, and (c) the effective 2PA cross-section vs input irradiance at $1200 \mathrm{~nm}$.

(Clark MXR, CPA 2010) is used to generate $1 \mathrm{~mJ}, 150 \mathrm{fs}$ (FWHM) pulses, with $1 \mathrm{kHz}$ repetition rate at $775 \mathrm{~nm}$ to pump an optical parametric amplifier (OPA, Light Conversion, TOPAS C) to produce wavelengths ranging between $1100 \mathrm{~nm}$ to $2600 \mathrm{~nm}$.

The Z-Scan technique allows for the absolute determination of the 2PA cross section $\left(\delta_{2 P A}\right)$. In addition to 2PA, ESA also contributes to NLA and the effect of each should be considered while characterizing the NLA of a material. Open aperture (OA) Z-Scan measurements on Aza-BODIPY dyes at $1200 \mathrm{~nm}$ are shown in Fig.2. The fitted results of measurements at 1200, 1500 and $1700 \mathrm{~nm}$ are shown in Table 1. At the wavelength of $1500 \mathrm{~nm}$ and $1700 \mathrm{~nm}$, data was fitted by assuming purely 2PA. The nonlinear transmission can be calculated by considering a pure 2PA mechanism and the irradiance is given by,

$$
\frac{d I}{d z}=-\alpha_{2} I^{2}
$$

where $I$ is the irradiance and $\alpha_{2}$ is the 2PA coefficient.

At the wavelength of $1200 \mathrm{~nm}$, ESA contributes to the signal that needs to be considered in the fitting. This can be done by considering the ESA cross-section $\left(\sigma_{E S A}\right)$, as follows

$$
\frac{d I}{d z}=-\alpha_{2} I^{2}-\sigma_{E S A} N I=-\alpha_{2, e f f}(I) I^{2}
$$

where $N$ is the number of population in the first excited state, assumed to be given by

$$
\frac{d N}{d t}=\frac{\alpha_{2} I^{2}}{2 \hbar \omega}
$$

Table 1. Nonlinear absorption coefficients of molecules studied in this work. 2PA cross section, $\delta_{2 P A}$, unit in Göppert-Mayer $(\mathrm{GM})$ defined as $10^{-50} \mathrm{~cm}^{4}$. $s \cdot$ molecule $^{-1} \cdot$ photon $^{-1}$. Excited-state absorption cross section, $\sigma_{E S A}$, unit in $10^{-20} \mathrm{~m}^{-2}$

\begin{tabular}{|l|c|c|c|c|c|}
\hline$\lambda(\mathrm{nm})$ & & SD238 & SD249 & SD190 & SD230 \\
\hline \multirow{2}{*}{1200} & $\delta_{2 P A}$ & $1900 \pm 200$ & $400 \pm 70$ & $280 \pm 50$ & $130 \pm 10$ \\
\cline { 2 - 6 } & $\sigma_{E S A}$ & $1.0 \pm 0.2$ & $3.5 \pm 0.8$ & $7.1 \pm 0.9$ & $2.0 \pm 0.3$ \\
\hline 1500 & $\delta_{2 P A}$ & $270 \pm 50$ & $250 \pm 50$ & $<50$ & $<50$ \\
\hline 1700 & $\delta_{2 P A}$ & $200 \pm 40$ & $260 \pm 60$ & $80 \pm 20$ & $65 \pm 15$ \\
\hline
\end{tabular}

It is also possible to define the irradiance dependent effective 2PA cross-section, as shown in Fig.2(c), where the intercept gives the pure 2PA and the slope gives the ESA crosssection. These values are close to values deduced from the fits considering each mechanism, reported in Table 1 for 1200 , 1500 and $1700 \mathrm{~nm}$

Extinction coefficients and two-photon spectra for AzaBODIPY dyes are presented in Fig.3. For comparison with the transition wavelength, spectra are plotted on the same graph with separate axes for extinction coefficient (bottom) and 2PA (top) photon wavelengths. As shown, at $1200 \mathrm{~nm}$ wavelength, both dye series have ESA. The brominated molecule, SD249, has a smaller 2PA cross section compared with SD238 and shows a 4-fold enhancement in ESA cross-section.

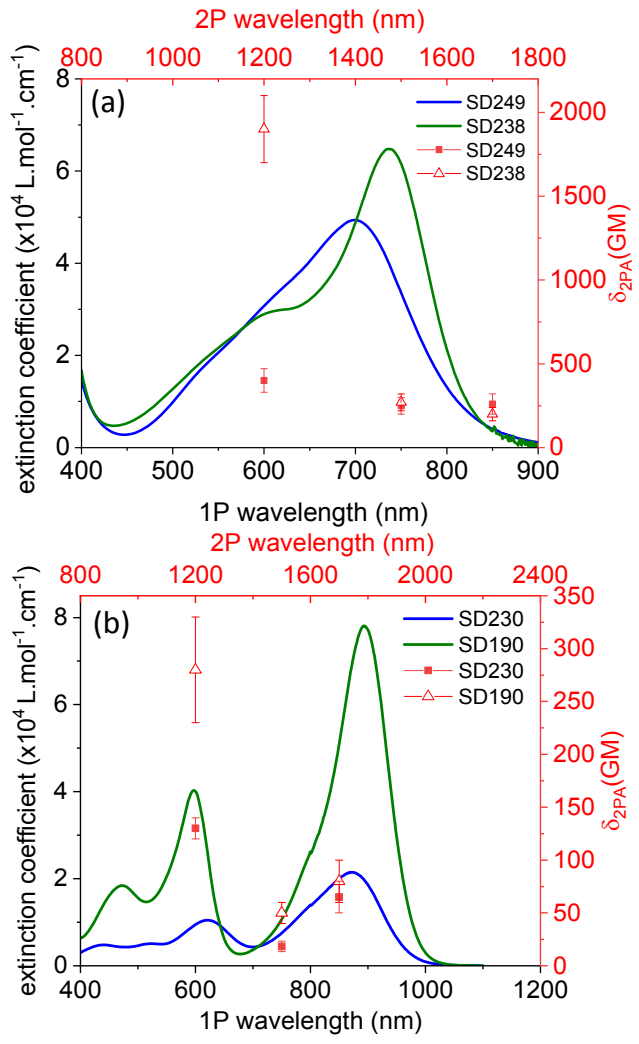

Fig.3. Extinction coefficient and 2PA coefficient of (a) SD249 and SD238, and (b) SD230 and SD190 dyes. 


\section{B. Conclusion}

We measured 2PA coefficients of Aza-BODIPY dyes at 1200,1500 , and $1700 \mathrm{~nm}$. At $1200 \mathrm{~nm}$, large 2PA coefficients and ESA cross-sections are observed and investigated in both dye series under intense ultrashort laser pulses. For the first series, we found a strong decrease in the 2PA cross-section for the brominated molecule and nearly a 4-fold enhancement of the ESA contribution at $1200 \mathrm{~nm}$. For the second Aza-BODIPY series bearing the benzothiadiazole moiety, we found a small 2PA cross-section at $1200 \mathrm{~nm}$ but with a large ESA crosssection.

\section{ACKNOWLEDGMENTS}

We gratefully thank Army Research Laboratory (ARL) (W911NF-15- 2-0090), National Science Foundation (NSF) (DMR-1609895), Air Force Office of Scientific Research (AFOSR) (FA9550-14-1-0040) MURI Center for Dynamic Magneto-Optics, and Thales Land and Air Systems (Thales LAS).

\section{REFERENCE}

1. Tutt, L.W. and T.F. Boggess, A review of optical limiting mechanisms and devices using organics, fullerenes, semiconductors and other materials.

Progress in quantum electronics, 1993. 17(4): p. 299338.

2. $\quad$ Bouit, P.-A., et al., Two-Photon Absorption-Related Properties of Functionalized BODIPY Dyes in the Infrared Range up to Telecommunication Wavelengths. Advanced Materials, 2009. 21(10-11): p. 1151-1154.

3. Château, D., et al., Efficient hybrid materials for optical power limiting at telecommunication wavelengths. Journal of Materials Chemistry C, 2014. 2(26): p. 5105-5110.

4. Sheik-Bahae, M., et al., Sensitive measurement of optical nonlinearities using a single beam. IEEE journal of quantum electronics, 1990. 26(4): p. 760769. 